\title{
Geometry-constraint-scan imaging for in-line phase contrast micro-CT
}

\author{
Jian $\mathrm{Fu}^{*}$, Guangyuan Yu and Dekai Fan \\ Research Center of Digital Radiation Imaging and Biomedical Imaging, Beijing University of \\ Aeronautics and Astronautics, Beijing 100191, People's Republic of China
}

\begin{abstract}
X-ray phase contrast computed tomography (CT) uses the phase shift that x-rays undergo when passing through matter, rather than their attenuation, as the imaging signal and may provide better image quality in soft-tissue and biomedical materials with low atomic number. Here a geometry-constraint-scan imaging technique for in-line phase contrast micro-CT is reported. It consists of two circular-trajectory scans with x-ray detector at different positions, the phase projection extraction method with the Fresnel free-propagation theory and the filter back-projection reconstruction algorithm. This method removes the contact-detector scan and the pure phase object assumption in classical in-line phase contrast Micro-CT. Consequently it relaxes the experimental conditions and improves the image contrast. This work comprises a numerical study of this technique and its experimental verification using a biomedical composite dataset measured at an x-ray tube source Micro-CT setup. The numerical and experimental results demonstrate the validity of the presented method. It will be of interest for a wide range of in-line phase contrast Micro-CT applications in biology and medicine.
\end{abstract}

Keywords: X-ray in-line phase contrast imaging, micro computed tomography, geometry-constraint scanning mode, reconstruction algorithm

\section{Introduction}

X-ray computed tomography $(\mathrm{CT})$ enables the non-destructive visualization of the internal structure of objects [1-5] and is a powerful analysis tool in biology and medicine [6-9]. In particular, for medical diagnostic applications, $\mathrm{x}$-ray $\mathrm{CT}$ has become an invaluable tool during the last 30 years. However, the conventional x-ray CT uses the intensity attenuation that x-rays undergo when passing through matter as the imaging signal and provides poor contrast and spatial resolution for weakly absorbing materials and structures with low atomic number. For example, since the absorption coefficients of soft tissue are very close to those of water, it is very difficult to distinguish internal features in the soft materials [6].

In order to produce better contrast of weakly absorbing materials and structures, $\mathrm{x}$-ray phase contrast $\mathrm{CT}$ (PC-CT) has been explored over the last years. The phase shift cross section is three orders of magnitude larger than the absorbing cross section for biomedical materials with low atomic number, which is

\footnotetext{
*Corresponding author: Jian Fu, Research Center of Digital Radiation Imaging and Biomedical Imaging, Beijing University of Aeronautics and Astronautics, Beijing 100191, People's Republic of China. Tel.:86-10-924339095; Fax: 86-10-82339095; E-mail: fujian706@buaa.edu.cn.
} 
an indication that PC-CT provides much better contrast resolution in soft tissue and low atomic samples than attenuation-contrast CT $[3,10,11]$. Several experimental case studies reported in the literature have demonstrated the validity of X-ray PC-CT techniques. There currently exist four kinds of PC-CT methods [1,12-29], which are based on the crystal interferometer, the analyzer, the grating interferometer and the free-propagation theory respectively [30]. The propagation-based PC-CT, also called in-line PC-CT, is in many senses the simplest kind of phase contrast imaging, as no optical elements are required in the beam and the constraint on the spectral width is relaxed [12,14,23,28]. It has a promising future on engineering applications.

In-line phase contrast micro-CT has been developed to get a spatial resolution in the micrometer range and has become a useful tool in the field of biomedical imaging [6, 12, 28, 31-33]. Phase projection scanning and extraction methods play a key role in these systems. Currently there are two kinds of phase projection scanning and extraction methods [31,34-38]. The first one needs two circular-trajectory scans $[31,34,35,38]$, one of which must be a contact-detector scan. It is sometimes impossible due to the constraint from the experimental system and the samples. The second one deletes the contactdetector scan but assumes that the specimen has no intensity attenuation to $\mathrm{x}$-ray and is a pure phase object $[6,31,36-38]$. Obviously in practice this assumption will degrade the imaging results.

Here a new in-line phase contrast Micro-CT imaging technique is reported for soft-tissue and biomedical materials with low atomic number. With two circular-trajectory scans at different positions, the new method mitigates the necessity of contact-detector scan and pure phase object assumption. The experimental results demonstrate the efficiency of the new technique in terms of improving image contrast under relaxed experimental conditions.

\section{Methods and materials}

A three-dimensional object can be described by a complex refractive index distribution $n(x, y, z)=$ $1-\delta(x, y, z)+i \beta(x, y, z)$, where $x, y$ and $z$ describe the coordinate system of the sample. In in-line phase contrast Micro-CT, one measures the projection images of the real part $\delta$ by evaluating the interference fringes arising in the free space propagation in the Fresnel regime and then reconstructs $\delta$ from the measured projection images.

\subsection{Geometry-constraint-scan mode}

The proposed geometry-constraint-scan mode is depicted in Figure 1. It is similar to the conventional Micro-CT circular trajectory scanning method. The difference is that this technique will execute two scans with x-ray detector at two positions $p_{1}$ and $p_{2}$. At each scan, the specimen is illuminated by an $\mathrm{x}$-ray source with a spot size in a micrometer range. X-ray detector acquires high-resolution projection images. The specimen is rotated over 360 degrees in a number of discrete steps, often several hundred, depending on the spatial resolution needed. At each angular position, the projection image is captured and stored by computer.

\subsection{Phase projection extraction method}

According to the Fresnel free propagation theory and the Taylor expansion, the intensity projection at the detector position $p_{1}$ in the case depicted in Figure 1 can be approximately expressed by

$$
I_{\theta}^{r_{1}}\left(M_{1} x, M_{1} y\right) \approx \frac{r_{0}^{2}}{\left(r_{0}+r_{1}\right)^{2}} \times I_{\theta}^{0}(x, y) \times\left[1-\frac{\lambda r_{1}}{2 \pi M_{1}} \nabla^{2} \phi_{\theta}(x, y)\right] .
$$




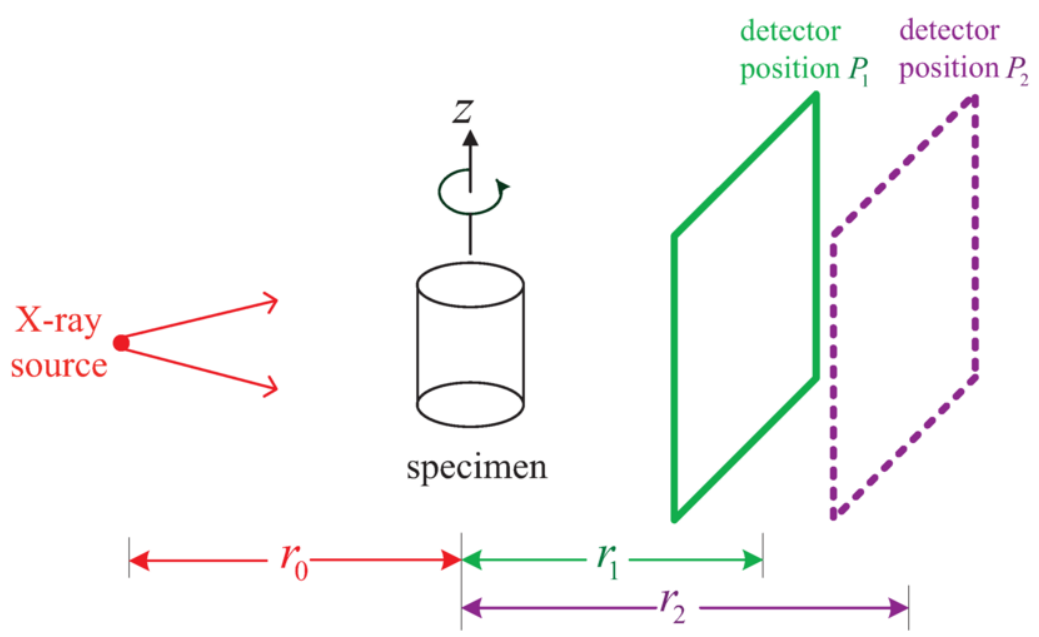

Fig. 1. Scanning configuration of geometry-constraint-scan mode in in-line phase contrast cone-beam Micro-CT. $z$ represents the rotation axis. $p_{1}$ and $p_{2}$ are the positions of x-ray detector. $r_{0}, r_{1}$ and $r_{2}$ are the distances among x-ray source, specimen and detector.

Where $\theta$ represents the rotation angle, $r_{0}$ and $r_{1}$ are the distance between the x-ray source and the specimen and the distance between the specimen and the detector respectively as shown in Figure $1, \lambda$ the x-ray wavelength, $M_{1}$ geometrical magnification factor, $t(x, y)$ the specimen function, $\nabla^{2}$ the Laplacian transform operator, $\phi_{\theta}(x, y)$ the phase projection and $I_{\theta}^{r_{1}}$ the detected intensity.

Similarly, the intensity projection at the detector position $p_{2}$ can be expressed by

$$
I_{\theta}^{r_{2}}\left(M_{2} x, M_{2} y\right) \approx \frac{r_{0}^{2}}{\left(r_{0}+r_{2}\right)^{2}} \times I_{\theta}^{0}(x, y) \times\left[1-\frac{\lambda r_{2}}{2 \pi M_{2}} \nabla^{2} \phi_{\theta}(x, y)\right] .
$$

Where $r_{2}$ is the distance between specimen and detector and $M_{2}$ is the geometrical magnification factor.

Eqs. (1) and (2) illustrate the linear relationship between the detected intensity projection images and the Laplacian of the phase projection. They are the projection imaging models of geometry-constraintscan in-line phase contrast Micro-CT. Based on Eqs. (1) and (2), a phase projection variant can be defined and expressed by

$$
g_{\theta}(x, y)=\frac{-\lambda}{2 \pi} \nabla^{2} \phi_{\theta}(x, y)=\frac{\left(r_{0}+r_{1}\right)^{2} \times I_{\theta}^{r_{1}}\left(M_{1} x, M_{1} y\right)-\left(r_{0}+r_{2}\right)^{2} \times I_{\theta}^{r_{2}}\left(M_{2} x, M_{2} y\right)}{r_{1} / M_{1} \times I_{\theta}^{r_{2}}\left(M_{2} x, M_{2} y\right)-r_{2} / M_{2} \times I_{\theta}^{r_{1}}\left(M_{1} x, M_{1} y\right)} .
$$

Where $g_{\theta}(x, y)$ represents the Laplacian projection at the angular position $\theta$. After taking twodimensional Fourier transform to Eq.(3), Eq.(4) can be obtained as

$$
F\left(\phi_{\theta}(x, y)\right)=\frac{1}{2 \pi \lambda\left(u^{2}+v^{2}\right)} F\left(g_{\theta}(x, y)\right) .
$$

Where $F$ represents the Fourier transform and $u$ and $v$ the frequency variants. Eq. (4) shows that the phase projection can be retrieved by two-dimensional filter operation to the intensity images recorded by the detector at two positions. 


\subsection{Image reconstruction algorithm}

Since the phase projection can be extracted with Eqs. (3) and (4), the following tomographic algorithm can be used to reconstruct the phase distribution of the specimen function for the case depicted in Figure 1

$$
f(x, y, z)=\frac{1}{4 \pi^{2}} F D K\left(F^{-1}\left(\frac{F\left(g_{\theta}(x, y)\right)}{u^{2}+v^{2}}\right)\right) .
$$

Where $F D K()$ represents the popular FDK algorithm [39] adopted in x-ray cone-beam CT and $F^{-1}$ the inverse Fourier transform.

The reconstruction procedure of the algorithm in Eq. (5) is described as follows. Firstly the phase projection variant $g_{\theta}(x, y)$ is calculated out by inputting the detected intensity images $I_{\theta}^{r_{1}}, I_{\theta}^{r_{2}}$ and the imaging parameters into Eq.(3). Then the phase projection is retrieved by filtering $g_{\theta}(x, y)$ using the filter $1 /\left(u^{2}+v^{2}\right)$. Finally FDK algorithm is applied to the retrieved phase projection to reconstruct the CT slices.

\section{Simulations}

Numerical simulations are presented in this section to demonstrate the proposed method. Reconstructions are performed for a 3D sphere phantom consisting of three spheres. The diameters are set to be $0.95 \mathrm{~cm}, 0.3 \mathrm{~cm}$ and $0.3 \mathrm{~cm}$ respectively. The phase indexes are $1 \times 10^{-10}, 2 \times 10^{-10}$ and 0 respectively.

The simulation parameters are listed here. The photon energy is $50 \mathrm{Kev}$ and the size of detector $2 \mathrm{~cm}$. The angular increment is $2^{\circ}$ and 180 rotation steps are adopted in $360^{\circ}$. The distances $r_{0}, r_{1}$ and $r_{2}$ are set to be $250 \mathrm{~mm}, 125 \mathrm{~mm}$ and $250 \mathrm{~mm}$, respectively.

Figure 2 displays the simulation results. Figures 2(a) and 2(b) are two of the simulated intensity projection images at positions $p_{1}$ and $p_{2}$, respectively. Figures 2(c) and 2(d) are the theoretical slice and the reconstructed slice using Eq. (5) respectively. Comparing Figure 2(d) with Figure 2(c), no disparity is found. It demonstrates the validity of the presented technique. Figure 2(e) gives out the grey value line profiles through the theoretical slice and the reconstructed slice indicated by the blue solid line in Figure 2(c) and the red dash line in Figure 2(d), and quantitatively supports this conclusion.

\section{Experiment}

The conclusions drawn from the numerical studies were verified experimentally by testing the scanning mode and the reconstruction algorithm with measured dataset. The specimen used for this test is a biomedical composite. The experimental dataset was recorded using a cone beam phase contrast MicroCT system with an x-ray tube source. The source size is $1 \mu \mathrm{m}$. The adopted x-ray detector consists of $2304 \times 2284$ channels with a channel size $50 \mu \mathrm{m}$. The experiment was performed at room temperature using the photon energies $80 \mathrm{Kev}$ and the tube current $240 \mathrm{~mA}$. The rotation angular increment is $0.375^{\circ}$ and 960 projections were recorded at each scanning.

The distances $r_{0}, r_{1}$ and $r_{2}$ are set to be $71 \mathrm{~mm}, 429 \mathrm{~mm}$ and $929 \mathrm{~mm}$ respectively. Figure 3 displays the experimental results. Figures 3(a) and (b) are two typical projection images recorded by detector at 


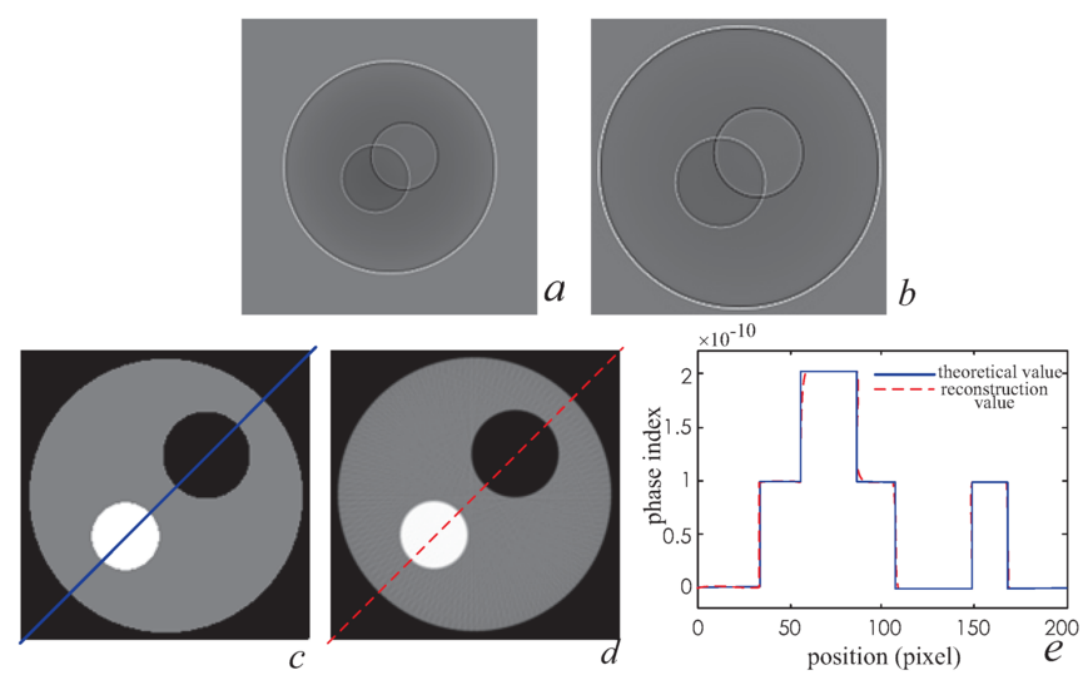

Fig. 2. Simulation results. (a) and (b) are two of the simulated intensity projection images at positions $p_{1}$ and $p_{2}$. (c) and (d) are the theoretical slice and the reconstructed slice using Eq. (5) respectively. (e) gives out the grey value line profiles through the theoretical slice and the reconstructed slice indicated by the blue solid line in (c) and the red dash line in (d).

two positions with different geometrical magnification. Figure 3(c) is one of the reconstructed CT slices using the conventional FDK algorithm. Figure 3(d) is one of the reconstructed CT slices using the new algorithm in Eq. (5). Obviously Figure 3(d) has a better contrast and less noise than Figure 3(c). It is helpful to recognize the internal structure details of the sample. These results demonstrate the validity of the proposed technique and the advantage over the conventional method.

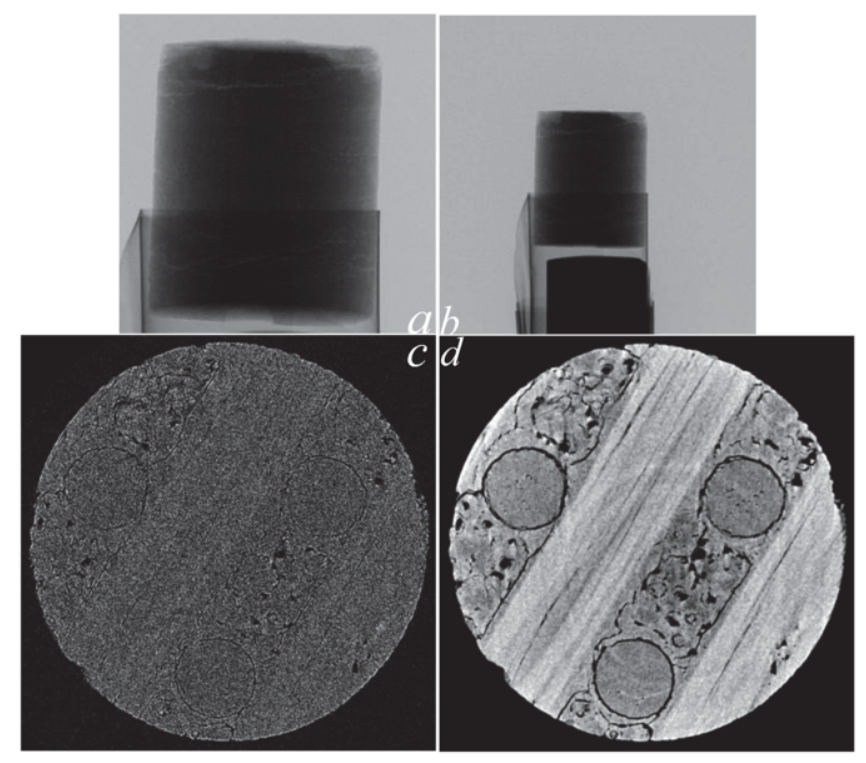

Fig. 3. Experimental results. (a) and (b) are two intensity projection images acquired by x-ray detector at two positions with different geometrical magnification. (c) and (d) are the reconstructed slice using the conventional FDK algorithm and the proposed method in Eq. (5) respectively. 


\section{Discussion and conclusion}

In this paper, a geometry-constraint-scanning imaging technique for in-line phase contrast cone beam Micro-CT is proposed and verified by numerical simulation and experiments. It consists of a scanning mode with geometrical constraints, a phase projection extraction method and the reconstruction algorithm. The scanning mode with geometrical constraints takes into account the absorbing effect of samples and makes a decisive contribution to the performance of this technique. It removes the pure phase object assumption of the conventional imaging method and is suitable for the sample with non-ignored absorption. We envisage that, particularly for biomedical imaging applications, where Micro-CT has been proven to be a useful method, this method will provide a possible resolution for some imaging requirements and push the future applications in biology and medicine.

\section{Acknowledgement}

This work was partly supported by the National Natural Science Foundation of China (Grant No. 11179009), Program for New Century Excellent Talents in University (NCET) from Ministry of Education of P.R.China and Beijing NOVA program (2009A09).

\section{References}

[1] C. Raven, A. Snigirev, I. Snigireva, P. Spanne, A. Souvorov and V. Kohn, Phase-contrast microtomography with coherent high-energy synchrotron x rays, Appl. Phys. Lett. 69 (1996),1826-1828.

[2] J. Fu, H. N. Lu, L. Zhang and J. J. Sun, X-CT imaging method for large objects using double offset scan mode, Nuclear Instruments and Methods in Physics Research Section A: Accelerators, Spectrometers, Detectors and Associated Equipment 575 (2007),519-523.

[3] F. Pfeiffer, C. David, O. Bunk, T. Donath, M. Bech, G. L. Duc, A. Bravin and P. Cloetens, Region-of-interest tomography for grating-based differential phase-contrast imaging, Phys. Rev.Lett. 101 (2008), 168101.

[4] J. Fu, B. H. Jiang, B. Li, P. Li and Q. L. Wang, Methods determining the angular increment of a continuous scan cone-beam CT system, IEEE Transactions on Nuclear Science 57 (2010),1071-1076.

[5] J. Fu, R. B. Tan, J. S. Deng and M. Liu, A cone beam computed tomography inspection method for fuel rod cladding tubes, Nuclear Instruments and Methods in Physics Research Section A: Accelerators, Spectrometers, Detectors and Associated Equipment 688 (2012),1-6.

[6] J. Fu and R. B. Tan, In-line phase contrast micro-CT reconstruction for biomedical specimens, Bio-Medical Materials and Engineering 24 (2014),431-437.

[7] J. Fu and L. Y. Chen, Single-slice reconstruction method for helical cone-beam differential phase-contrast CT, Bio-Medical Materials and Engineering 24 (2014),45-51.

[8] J. Fu, M. Willner, L. Chen, R. Tan, K. Achterhold, M. Bech, J. Herzen, D. Kunka, J. Mohr and F. Pfeiffer, Helical differential X-ray phase-contrast computed tomography, Phys. Med. 30 (2014),374-379.

[9] J. Fu, R. B. Tan and L. Y. Chen, Analysis and accurate reconstruction of incomplete data in X-ray differential phasecontrast computed tomography, Anal. Bioanal. Chem. 406 (2014),897-904.

[10] R. Fitzgerald, Phase-sensitive x-ray imaging, Phys. Today 7 (2000),23-27.

[11] A. Momose, Recent advances in x-ray phase imaging, Jpn. J. Appl. Phys. 44 (2005),6355-6359.

[12] S. W. Wilkins, T. E. Gureyev, A. Pogany and A. W. Stevenson, O. Bunk and C. David, Phase-contrast imaging using polychromatic hard x-rays, Nature 384 (1996), 335-337.

[13] F. Beckmann, K. Heise, B. Kolsch, U. Bonse, M. Rajewsky and T. Biermann, Three-dimensional imaging of nerve tissue by x-ray phase-contrast microtomography, Biophys. J. 76 (1999),98-102.

[14] P. Cloetens, W. Ludwig, J. Baruchel, D. van Dyck, J. van Landuyt, J. P. Guigay and M. Schlenker, Holotomography:quantitative phase tomography with micrometer resolution using hard synchrotron radiation x rays, Appl. Phys. Lett. 75 (1999),2912-2924.

[15] F. A. Dilmanian, Z. Zhong, B. Ren, X. Y. Wu, L. D. Chapman, I. Orion and W. C. Thomlinson, Computed tomography of x-ray index of refraction using the diffraction enhanced imaging method, Phys. Med.Biol. 45 (2000), 933-946. 
[16] S. C. Mayo, T. J. Davis, T. E. Gureyev, P. R. Miller, D. Paganin, A. Pogany, A. W. Stevenson and S. W. Wilkins, X-ray phase-contrast microscopy and microtomography, Opt. Express 11 (2003),2289-2302.

[17] P. J. McMahon, A. G. Peele, D. Paterson, J. J. A. Lin, T. H. K. Irving, I. McNulty and K. A. Nugent, Quantitative x-ray phase tomography with sub-micron resolution, Opt. Commun. 217 (2003),53-57.

[18] A. Momose, S. Kawamoto, I. Koyama, Y. Hamaishi, K. Takai and Y. Suzuki, Demonstration of x-ray Talbot interferometry, Jpn. J. Appl. Phys. 42 (2003),L866-L868.

[19] T. Weitkamp, A. Diaz, C. David, F. Pfeiffer, M. Stampanoni, P. Cloetens and E. Ziegler, X-ray phase imaging with a grating interferometer, Opt. Express 13 (2005),6296-6304.

[20] A. Groso, M. Stampanoni, R. Abela, P. Schneider, S. Linga and R. Müller, Phase contrast tomography: an alternative approach, Appl. Phys. Lett. 88 (2006), 214104.

[21] A. Momose, W. Yashiro, Y. Takeda, Y. Suzuki and T. Hattori, Phase tomography by x-ray Talbot interferometry for biological imaging, Jpn. J. Appl. Phys. 45 (2006), 5254-5262.

[22] A. Bravin, J. Keyrilainen, M. Fernandez, S. Fiedler, C. Nemoz, M. L. Karjalainen-Lindsberg, M. Tenhunen, P. Virkkunen, M. Leidenius, K. Von Smitten, P. Sipila and P. Suortti, High-resolution CT by diffraction-enhanced x-ray imaging: mapping of breast tissue samples and comparison with their histo-pathology, Phys. Med. Biol. 52 (2007), 2197-2211.

[23] W. X. Cai and R. L. Ning, Design and construction of a micro-focus in-line phase-contrast cone-beam CT (PC-CBCT) system for soft tissue imaging, Proc. SPIE 7622, (2010), 76225F.

[24] J. Fu, P. Li, Q. L. Wang, S. Y. Wang, M. Bech, A. Tapfer, D. Hahn and F. Pfeiffer, A reconstruction method for equidistant fan beam differential phase contrast computed tomography, Phys. Med. Biol. 56, (2011), 4529-4538.

[25] J. Fu, A. Velroyen, R. B. Tan, J. W. Zhang, L. Y. Chen, A. Tapfer, M. Bech and F. Pfeiffer, A reconstruction method for cone-beam differential x-ray phase-contrast computed tomography, Optics Express 20, (2012), 21512-21519.

[26] J. Fu, S. Schleede, R. Tan, L. Chen, M. Bech, K. Achterhold, M. Gifford, R. Loewen, R. Ruth and F. Pfeiffer, An algebraic iterative reconstruction technique for differential x-ray phase-contrast computed tomography, Z. Med. Phys. 23, (2013), 186-193.

[27] A. Tapfer, M. Bech, A. Velroyen, J. Meiser, J. Mohr, M. Walter, J. Schulz, B. Pauwels, P. Bruyndonckx, X. Liu, A. Sasov and F. Pfeiffer, Experimental results from a preclinical x-ray phase-contrast ct scanner, Proc. Natl Acad. Sci. USA 109, (2012), 15529-15530.

[28] S. C. Mayo , A. W. Stevenson and S. W. Wilkins, In-line phase-contrast x-ray imaging and tomography for materials science, Proc. Natl Acad. Sci. USA 5, (2012), 937-965.

[29] T. Shimura, N. Morimoto, S. Fujino, T. Nagatomi, K. C. Oshima, J. Harada, K. O. K, N. Osaka, T. Hosoi and H. Watanabe, Hard x-ray phase contrast imaging using a tabletop talbot-lau interferometer with multiline embedded x-ray targets, Opt. Lett. 38, (2013), 157-159.

[30] J. Fu, Phase Contrast Computed Tomography, Computed Tomography - Clinical Applications, L. Saba, ed., InTech, Croatia, 2012.

[31] Y. D. Witte, Improved and practically feasible reconstruction methods for high resolution X-ray tomography, Ph.D. Dissertation, Universiteit Gent, 2010.

[32] Y. Xi, R. Tang, Y. Wang and J. Zhao, Microbubbles as contrast agent for in-line X-ray phase-contrast imaging, Applied Physics Letters 99, (2011), 011101.

[33] Q. Tao, D. Li, L. Zhang and S. Luo, Using x-ray in-line phase-contrast imaging for the investigation of nude mouse hepatic tumors, PLoS ONE 7, (2012), e39936.

[34] F. B. Meng, A. M. Yan, G. Zhou, X. Z. Wu and H. Liu, Development of a dual-detector X-ray imaging system for phase retrieval study, Nuclear Instruments Methods in Physics Research Section B-Beam Interactions with Materials and Atoms 254, (2007), 300-306.

[35] M. Langer, P. Cloetens, J. P. Guigay and F. Peyrin, Quantitative comparison of direct phase retrieval algorithms in in-line phase tomography, Med. Phys. 35, (2008), 4556-4566.

[36] M. N. Boone, W. Devulder, M. Dierick, L. Brabant, E. Pauwels, L. Van Hoorebeke, Comparison of two single-image phase-retrieval algorithms for in-line x-ray phase-contrast imaging, J Opt Soc Am A Opt Image Sci Vis 29, (2012), 26672672 .

[37] A. Groso, R. Abela, and M. Stampanoni, Implementation of a fast method for high resolution phase contrast tomography, Optics Express 14, (2006), 8103-8110.

[38] B. Li, Study of Theory on In-line Phase Contrast Imaging with Micro-focus X-ray Source, Ph.D. Dissertation, Beijing University of Aeronautics and Astronautics, 2011.

[39] L. A. Feldkamp, L. C. Davis and J. W. Kress, Practical cone-beam algorithm, J. Opt. Soc. Am. A 1, (1984),612-619. 